\title{
Marked Reduction of the Number and Individual Volume of Sebaceous Glands in Psoriatic Lesions
}

\author{
Aikaterini I. Liakou ${ }^{a}$ Jens R. Nyengaard ${ }^{c} \quad$ Stefanos Bonovas $^{d} \quad$ Juergen Knolle ${ }^{b}$

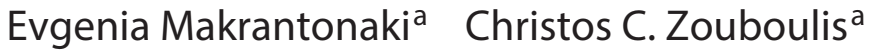 \\ a Departments of Dermatology, Venereology, Allergology and Immunology, and ${ }^{\mathrm{b}}$ Institute of Pathology, \\ Dessau Medical Center, Dessau, Germany; ' Department of Clinical Medicine, Stereological Research Laboratory, \\ Aarhus, Denmark; ${ }^{d}$ Humanitas Clinical and Research Center, Milan, Italy
}

\section{Key Words \\ Psoriasis · Alopecia · Psoriatic alopecia · Sebaceous gland}

\begin{abstract}
Background: Psoriasis is a chronic inflammatory skin disease characterized by plaques with inflammation, infiltration, hyper-/parakeratosis and desquamation. Microscopic findings in previous studies have revealed some degree of atrophy of the sebaceous glands in patients with psoriasis vulgaris and psoriatic alopecia. Objective: The aim of this study was to investigate possible changes of the sebaceous glands in patients with psoriatic plaques and especially psoriatic alopecia. Methods: Histological and stereological analyses were performed in skin specimens from involved and healthylooking skin of 14 patients with psoriasis. Stereology detects and quantifies 3-dimensional structures ex vivo. Furthermore, the differentiation process of sebocytes of another 14 psoriatic patients was examined by immunohistochemistry of involved and uninvolved skin specimens. Results: A significant reduction of the number of sebaceous glands as well as of the volume of individual sebaceous glands was assessed in the lesional compared to the nonlesional psoriatic
\end{abstract}

skin. Moreover, it was likely that sebocytes in psoriatic lesions may not differentiate properly. Conclusion: These findings indicate that the sebaceous gland may be a player and not an innocent bystander in the development of psoriatic lesions and especially of psoriatic alopecia.

(c) 2016 S. Karger AG, Basel

\section{Introduction}

Psoriasis has a multifactorial pathogenesis. The main pathological features are the increased proliferation of keratinocytes and their faster but abnormal maturation. During the last 25 years, skin appendages have been proposed to also play a role or undergo some changes in psoriasis. In 1989, Headington et al. [1] observed sebaceous gland atrophy and decreased hair shaft diameters without alopecia in psoriatic scalp lesions. In 1994, Wilson et al. [2] confirmed the atrophy of sebaceous glands in psoriatic lesions of the body and the scalp, which was further confirmed by Werner et al. [3] in 2008. In 2012, Silva et al. [4] reported a $60 \%$ absence and $25 \%$ atrophy of the sebaceous glands in psoriatic lesions.

\section{KARGER}

E-Mail karger@karger.com

www.karger.com/drm
C 2016 S. Karger AG, Basel

$1018-8665 / 16 / 2324-0415 \$ 39.50 / 0$
Prof. Dr. med. Christos C. Zouboulis

Departments of Dermatology, Venereology, Allergology and Immunology Dessau Medical Center, Auenweg 38

DE-06847 Dessau (Germany)

E-Mail christos.zouboulis@ klinikum-dessau.de 
Fig. 1. Summary of the main differentiation markers of sebocytes and the stages of their expression. Blimp-1 may be both an early and terminal differentiation marker. There are markers (at the bottom of the figure) that may be expressed during the whole differentiation process of sebocytes. PPAR- $\gamma$ supports the differentiation of early to mature sebocytes, and PPAR- $\beta / \delta$ supports the differentiation of mature to late sebocytes.

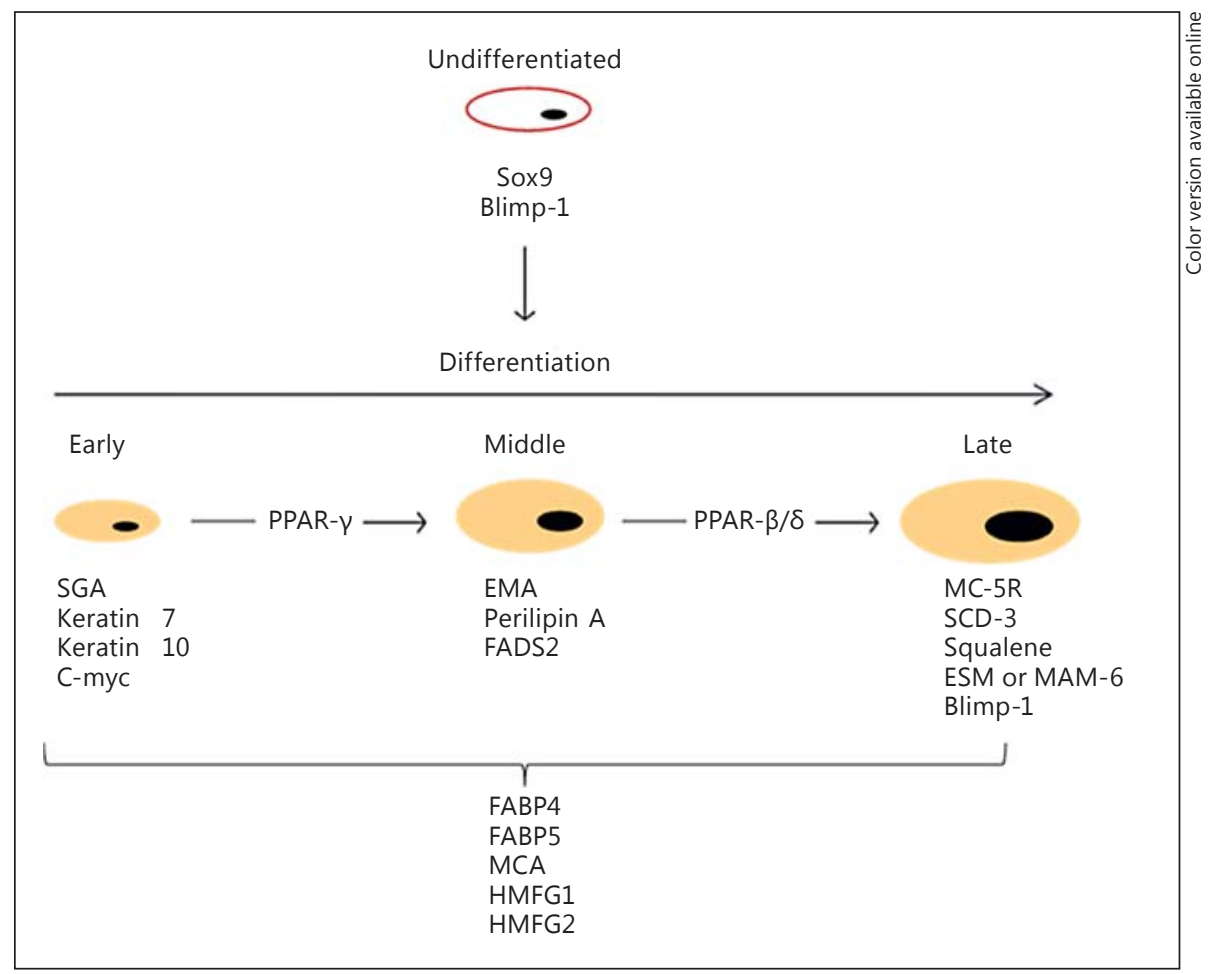

The major question raised through the years was whether there is a difference in the clinical and histopathological findings between those psoriatic patients who present alopecia and those who do not develop alopecia. Interestingly, the latest studies have shown that a large majority of patients with psoriatic alopecia have atrophy or complete absence of sebaceous glands at the time of presentation $[1,5]$.

The sebaceous gland and hair follicle constitute the pilosebaceous unit of the skin. During late embryogenesis, developing hair follicles display several bulges (hair peg stage), one of which gives rise to the sebaceous gland and is located just above the hair follicle stem cell bulge and below the infundibulum of the developing follicle [6]. The sebaceous gland arises as an outgrowth of the outer root sheath of the hair follicle, while undifferentiated sebocytes emerge from peripheral basal cells and then move centrally as (first partially and later fully) differentiated sebocytes. Differentiated sebocytes produce and secrete lipid-rich sebum into the hair canal that empties to the skin surface [6-10].

Sebaceous differentiation is accompanied by differentiation stage-characteristic protein patterns. Figure 1 summarizes the main differentiation markers of sebocytes and their stages of expression. Finally, sebocytes have been suggested to become, under certain circumstances, subject to a kind of 'keratinization', i.e. express markers of keratinocytes and lose their ability to produce sebum [11-13]. This theory may effectively explain the observed changes of the sebaceous glands in psoriasis and is supported by the fact that sebocytes and keratinocytes derive from the same progenitor cells [14].

\section{Materials and Methods}

The study plan and the demographic and clinical data of the patients are summarized in figure 2 and table 1 . The biopsy localizations are summarized in table 2.

A qualitative histological study of these biopsies was conducted to describe the presence or absence of the sebaceous glands and their number, distribution, and morphology (shape and size) in lesional and nonlesional skin. The volume of the sebaceous glands was estimated by the Cavalieri principle.

For the immunohistological study, biomarkers which recognize different differentiation stages of human sebocytes were applied. Table 3 summarizes the main antibodies used for immunohistochemical analysis.

For further details, see the supplementary materials (for all online suppl. material, see www.karger.com/doi/10.1159/000445942) (fig. 2; tables 1-3). 


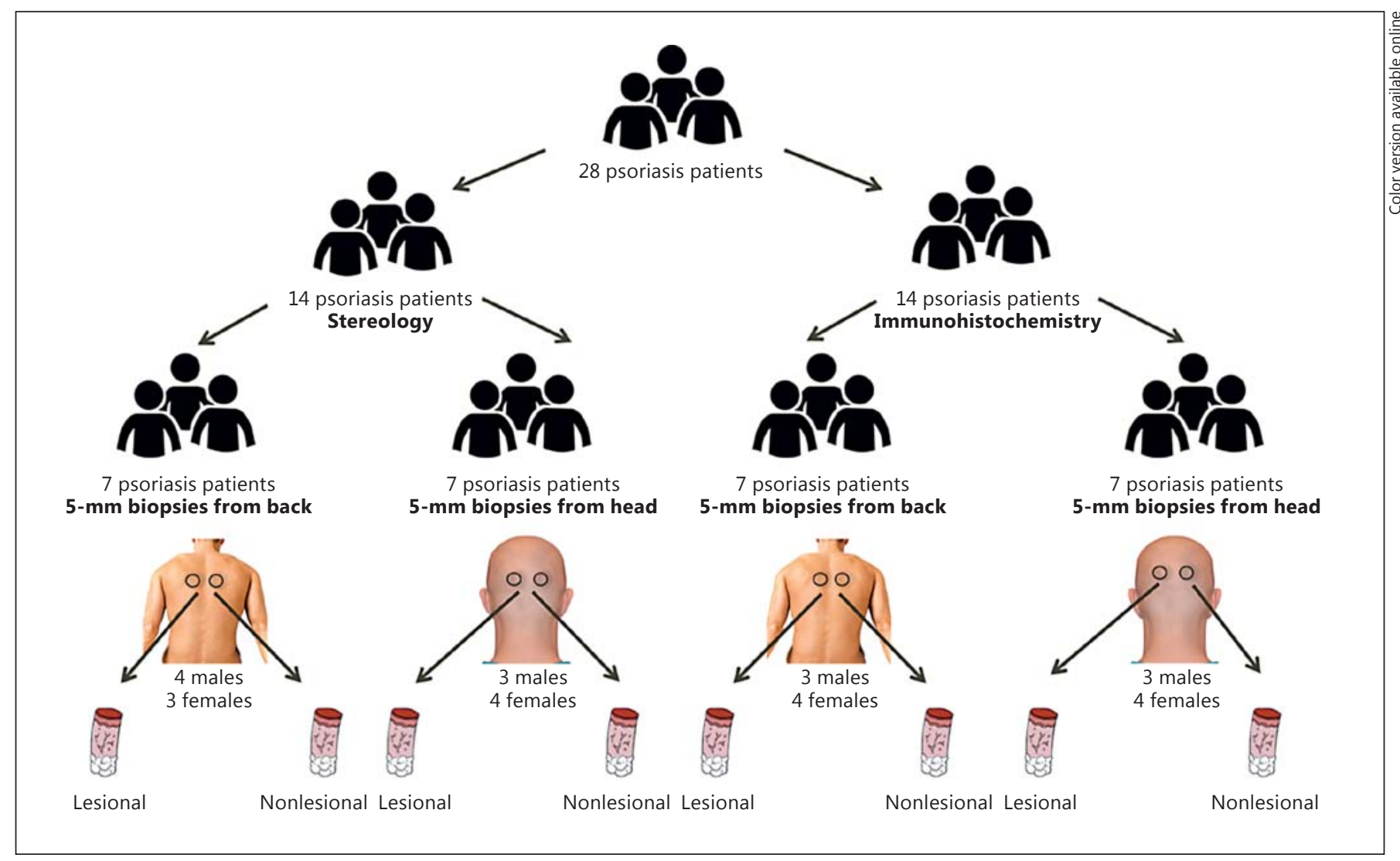

Fig. 2. Flowchart of Materials and Methods. Methodology of the study. The head and back areas were chosen as being among the most seborrheic areas of the human body, with a high frequency of sebaceous glands. A qualitative histological study of these biop- sies was conducted to describe the presence or absence of the sebaceous glands and their number, distribution, and morphology (shape and size) in lesional and nonlesional skin.

Table 1. Characteristics of the study population $(n=14)$

\begin{tabular}{lccc}
\hline & Mean \pm SD & Median (IQR) & $\mathrm{n}(\%)$ \\
\hline Age, years & $53.1 \pm 12.8$ & $53.5(45-61)$ & $7(50.0)$ \\
Male gender & & & \\
Sebaceous gland volume (psoriatic skin), $\mathrm{mm}^{3}$ & $0.018 \pm 0.030$ & $0.004(0.001-0.024)$ & \\
Sebaceous gland volume (healthy skin), $\mathrm{mm}^{3}$ & $0.057 \pm 0.053$ & $0.035(0.027-0.073)$ \\
Thickness of psoriatic epidermis, mm & $332.7 \pm 92.8$ & $332.0(260.0-400.7)$ \\
Thickness of healthy epidermis, mm & $80.5 \pm 22.3$ & $80.9(61.4-94.0)$ \\
\hline
\end{tabular}

\section{Results}

\section{Histology}

Conducting estimates of the volume of the sebaceous glands, 3 of the 14 patients were found to have no visible gland tissue in the lesional skin, although there were def- initely sebaceous glands in biopsies of their nonlesional skin. From the biopsies of the 11 patients who had visible gland tissue in the lesional skin, 5 originated from the back and 6 from the scalp. Figure 3 demonstrates the obvious absence or shrinkage of sebaceous glands in the lesional skin. 
Table 2. Summary of the clinical data on the patients who were recruited for the stereological and immunohistochemical analysis

\begin{tabular}{|c|c|c|c|c|}
\hline Patient No. & Gender & Age, years & Localization & PASI score \\
\hline \multicolumn{5}{|l|}{ Stereology } \\
\hline p1 & M & 35 & back & 11.4 \\
\hline p2 & M & 49 & back & 15.8 \\
\hline p3 & $\mathrm{F}$ & 64 & back & 17.6 \\
\hline p4 & $\mathrm{F}$ & 70 & back & 17.0 \\
\hline p5 & M & 36 & back & 9.0 \\
\hline p6 & $\mathrm{F}$ & 51 & back & 15.8 \\
\hline p7 & M & 61 & back & 14.0 \\
\hline p8 & $\mathrm{F}$ & 55 & head & 16.0 \\
\hline p9 & M & 45 & head & 18.8 \\
\hline p10 & $\mathrm{F}$ & 52 & head & 8.2 \\
\hline p11 & M & 33 & head & 19.6 \\
\hline p12 & $\mathrm{F}$ & 75 & head & 21.0 \\
\hline p13 & M & 59 & head & 20.2 \\
\hline p14 & $\mathrm{F}$ & 59 & head & 7.2 \\
\hline \multicolumn{5}{|c|}{ Immunohistochemistry } \\
\hline p1 & $\mathrm{M}$ & 49 & head & 15.8 \\
\hline p2 & $\mathrm{M}$ & 60 & head & 11.8 \\
\hline p3 & $\mathrm{F}$ & 67 & head & 24.3 \\
\hline p4 & $\mathrm{M}$ & 63 & head & 12.2 \\
\hline p5 & $\mathrm{F}$ & 39 & head & 7.0 \\
\hline p6 & $\mathrm{F}$ & 64 & head & 6.0 \\
\hline p7 & $\mathrm{F}$ & 69 & head & 4.2 \\
\hline p8 & $\mathrm{F}$ & 65 & back & 15.8 \\
\hline p9 & $\mathrm{F}$ & 55 & back & 24 \\
\hline p10 & $\mathrm{M}$ & 73 & back & 20.2 \\
\hline p11 & $\mathrm{M}$ & 54 & back & 30.6 \\
\hline p12 & $\mathrm{F}$ & 30 & back & 12.4 \\
\hline p13 & $\mathrm{M}$ & 45 & back & 21.8 \\
\hline p14 & $\mathrm{F}$ & 75 & back & 21.0 \\
\hline
\end{tabular}

PASI $=$ Psoriasis Area and Severity Index.

\section{Stereological Volume Estimation}

The volume of the sebaceous gland tissue is shown in figure 4 , and the mean volume in lesional skin was on average approximately $20 \%$ of that of healthy controls ( 0.018 vs. $\left.0.057 \mathrm{~mm}^{3} ; \mathrm{p}=0.001\right)$. When only considering the biopsies in which both lesional and nonlesional skin contained sebaceous gland tissue (fig. 5), the volume of sebaceous gland tissue was approximately $25 \%$ of that of healthy controls ( 0.023 vs. $\left.0.062 \mathrm{~mm}^{3} ; \mathrm{p}=0.003\right)$. Figure 6 shows schematically, in a point diagram, the differences in sebaceous gland volume between lesional and nonlesional skin. The differences in and the statistical significance of sebaceous gland volume between male and female patients between the back and the head are summarized in online supplementary table 1.
Table 3. The antibodies that were used for the immunohistochemistry and their dilution and pretreatment conditions, after being several times titrated and tested in all possible conditions

\begin{tabular}{lll}
\hline Antibody & Dilution & Pretreatment \\
\hline Ki67 & $1: 400$ & pH 6.1 \\
CK7 & $1: 1,000$ & pH 9 \\
EMA & $1: 2,000$ & pH 6.1 \\
ESM & $1: 1,000$ & none \\
Involucrin & $1: 100$ & pH 6.1 \\
Negative control & $1: 100$ & none \\
\hline
\end{tabular}

\section{Stereological Estimation of the Epidermis Thickness}

The mean epidermis thickness of the lesional skin was increased compared to the nonlesional skin (online suppl. table 1). There was a statistically significant strong inverse correlation between epidermis thickness and sebaceous gland volume, as shown in the online supplementary table 2 . The results suggest that there is a positive correlation between the activity of the disease and the atrophy of the sebaceous glands - the more active the disease was the more atrophic sebaceous glands were detected.

\section{Immunohistochemistry}

Out of the 14 patients, 1 showed a clear difference between lesional and nonlesional skin with a stronger expression of the early sebaceous differentiation marker cytokeratin 7 (CK7), the advanced sebaceous differentiation marker epithelial membrane antigen (EMA), and the late differentiation marker epithelial sialomucin (ESM) in the lesional skin (fig. 7). Two patients showed a slight difference with CK7, EMA, and ESM being again more strongly expressed in the lesional compared to the nonlesional skin. The rest of the patients (11/14) showed no obvious difference between lesional and nonlesional skin (online suppl. table 3). The results suggest a possible tendency of sebocytes to be less differentiated in the lesional skin.

One of the 14 patients, the same patient mentioned above, showed a stronger expression of the proliferation marker Ki67 in the lesional skin than in the nonlesional skin (fig. 8). The rest of the patients (13/14) showed no obvious difference between lesional and nonlesional skin (online suppl. table 3 ). The results are controversial, since a higher rate of proliferation in psoriatic skin is widely known.

No differences were found between lesional and nonlesional skin regarding the expression of the marker of keratinocyte differentiation involucrin (fig. 8; online suppl. table 3).
Liakou/Nyengaard/Bonovas/Knolle/ Makrantonaki/Zouboulis 


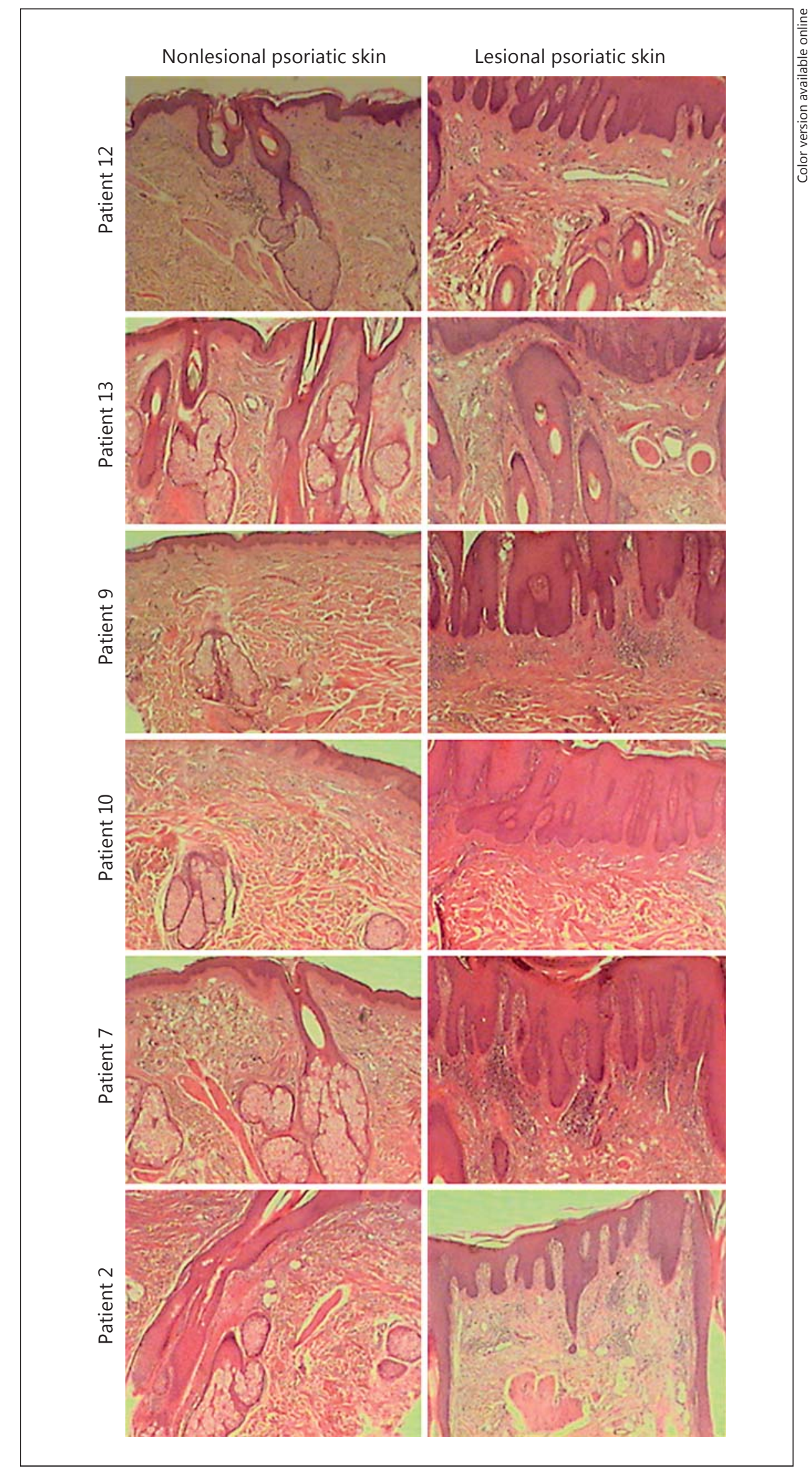

Fig. 3. Examples of the absence and shrinkage of sebaceous gland tissue in the lesional compared to the nonlesional psoriatic skin. 


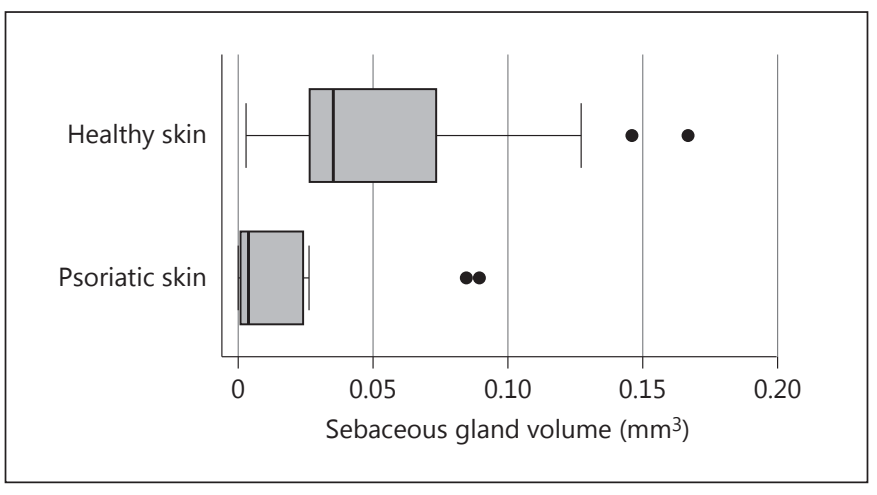

Fig. 4. Comparison of sebaceous gland volume between healthy and psoriatic skin $(\mathrm{n}=14)$. Results are presented with box-andwhisker plots. The sebaceous gland volume was significantly lower in psoriatic skin $(\mathrm{p}=0.001)$.

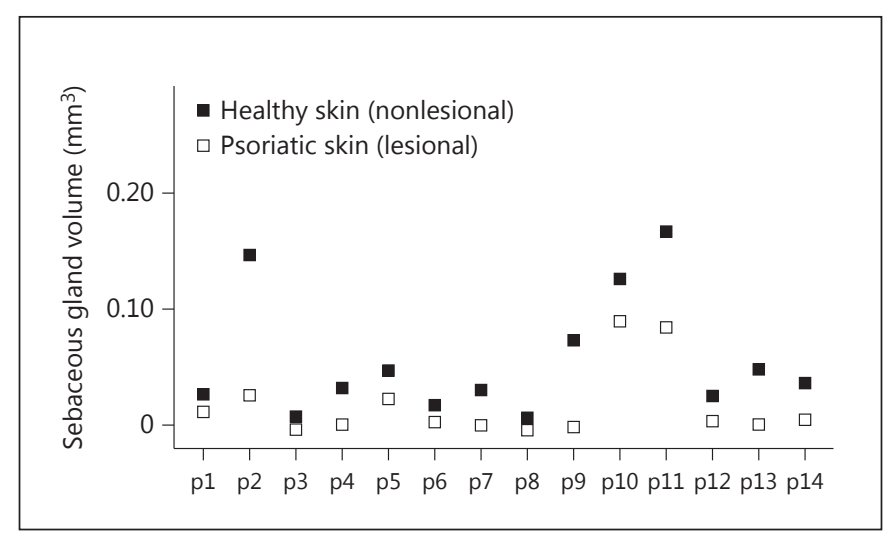

Fig. 6. Schematic presentation of the differences in sebaceous gland volume between psoriatic (lesional) and healthy (nonlesional) skin in psoriasis patients. $\mathrm{p} 1=$ Patient 1 , etc. The sebaceous glands are smaller in lesional psoriatic skin in almost all patients.

\section{Discussion}

The results of this study clearly demonstrate a reduction of sebaceous gland tissue volume in the lesional skin of psoriasis patients, confirming previous studies [1-4]. This finding is compatible with common clinical experience, since it is well known that the psoriatic plaque is characterized by thickness, dryness, and scaling, which are major clinical findings of sebostasis. The question which arises, namely 'does this finding indicate a role of sebaceous gland changes in the pathogenesis of psoriasis?', belongs to the hen and the egg principle, i.e. do pathological sebaceous glands cause the reactive thickness and

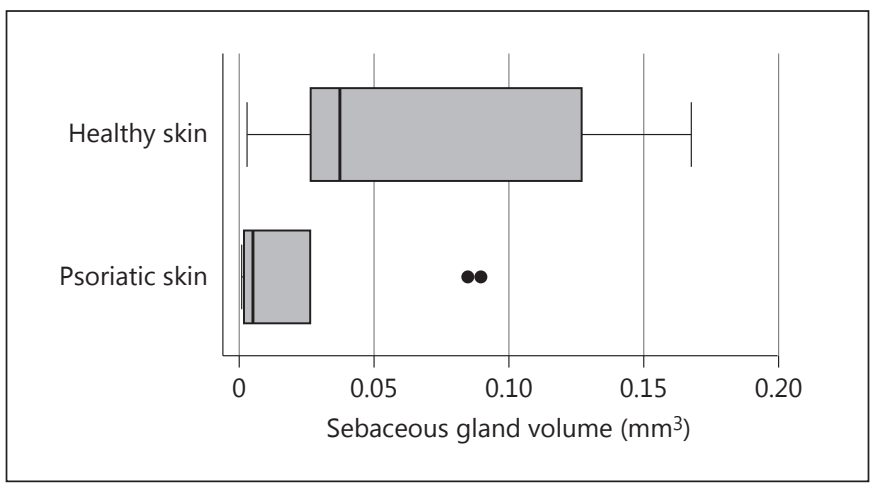

Fig. 5. Comparison of sebaceous gland volume between healthy and psoriatic skin $(n=11)$. Results are presented with box-andwhisker plots. The sebaceous gland volume was significantly lower in psoriatic skin $(\mathrm{p}=0.003)$.

dryness of the epidermis or is this reduction of sebaceous glands an epiphenomenon of the epithelial pathology.

In this study, four major differentiation markers of sebocytes were used. These markers have been mentioned in previous studies as possible or definite differentiation markers of sebocytes in vitro and ex vivo. However, this study definitely adds to these contributions by clearly showing the function of these four differentiation markers, which provides a useful tool in the study of sebaceous differentiation in the future. It should be mentioned that CK7 is presented as a definite early differentiation marker and ESM as a definite late differentiation marker [7, 15-17]. Regarding EMA, several opinions suggest that this antigen is expressed by all sebocytes during the entire differentiation process, so it cannot be exactly distinguished from the other two differentiation markers [7, 16]. This means, practically, that sebocytes in all three differentiation stages may express EMA, including the ones that express CK7 or ESM. Thus, while this study cannot definitely suggest that EMA is expressed in a certain differentiation stadium only, it suggests that it is expressed either by all sebocytes or by the advanced differentiated sebocytes. Furthermore, a discrimination of the continuous differentiation process of sebocytes between three stages was made for the needs of this study; some previous studies have made up to five-stage discrimination [7]. Ki67 did not reveal any problems; it is a known proliferation marker used to visualize the proliferation of sebocytes in the basal layer. Sebocytes can proliferate and differentiate concomitantly; only undifferentiated sebocytes mostly proliferate, whereas mature ones only differentiate [18]. 
Fig. 7. CK7 as an early differentiation marker was more strongly expressed in the lesional skin of patient 3. EMA and ESM as middle and late differentiation markers were more strongly expressed in the nonlesional skin of the same patient. This patient shows a lack of mature sebaceous differentiation in the lesional psoriatic skin, a finding that could explain the reduced sebum production at the psoriatic lesion.
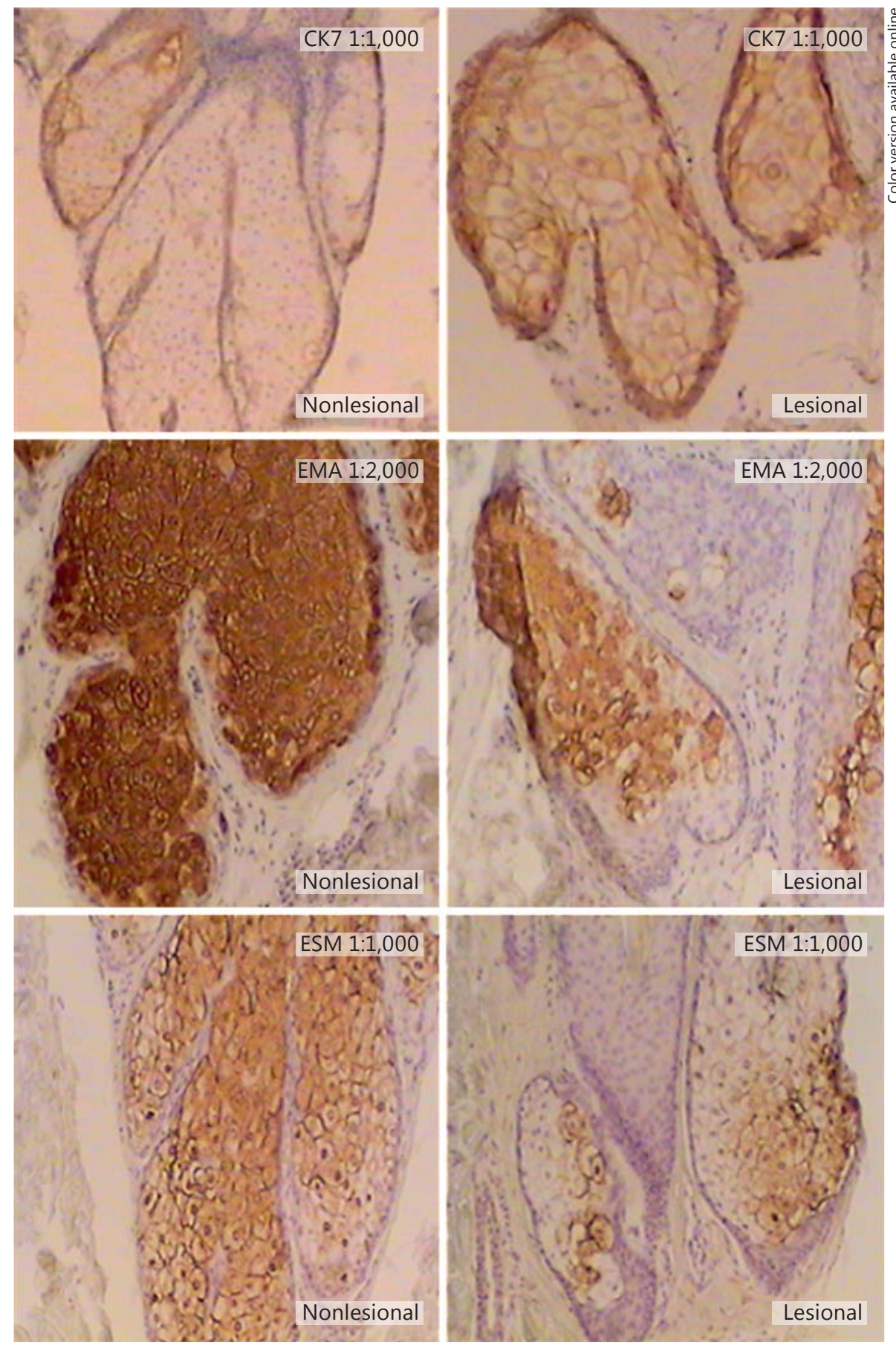

A further hypothesis was that the abnormal differentiation process of sebocytes in psoriatic lesions may represent a squamous keratinization able to induce inflammation, as shown in experimental settings $[13,19]$. It is well established that sebocytes are highly specialized, sebum-producing epithelial cells that release their content by rupture of the cell membrane and cellular degradation with the function of holocrine secretion. Sebocytes are most commonly found in the skin in association with hair follicles (forming the pilosebaceous unit) where they arise from undifferentiated hair follicle epithelial cells. Sebum forms an integral component of the epidermal 


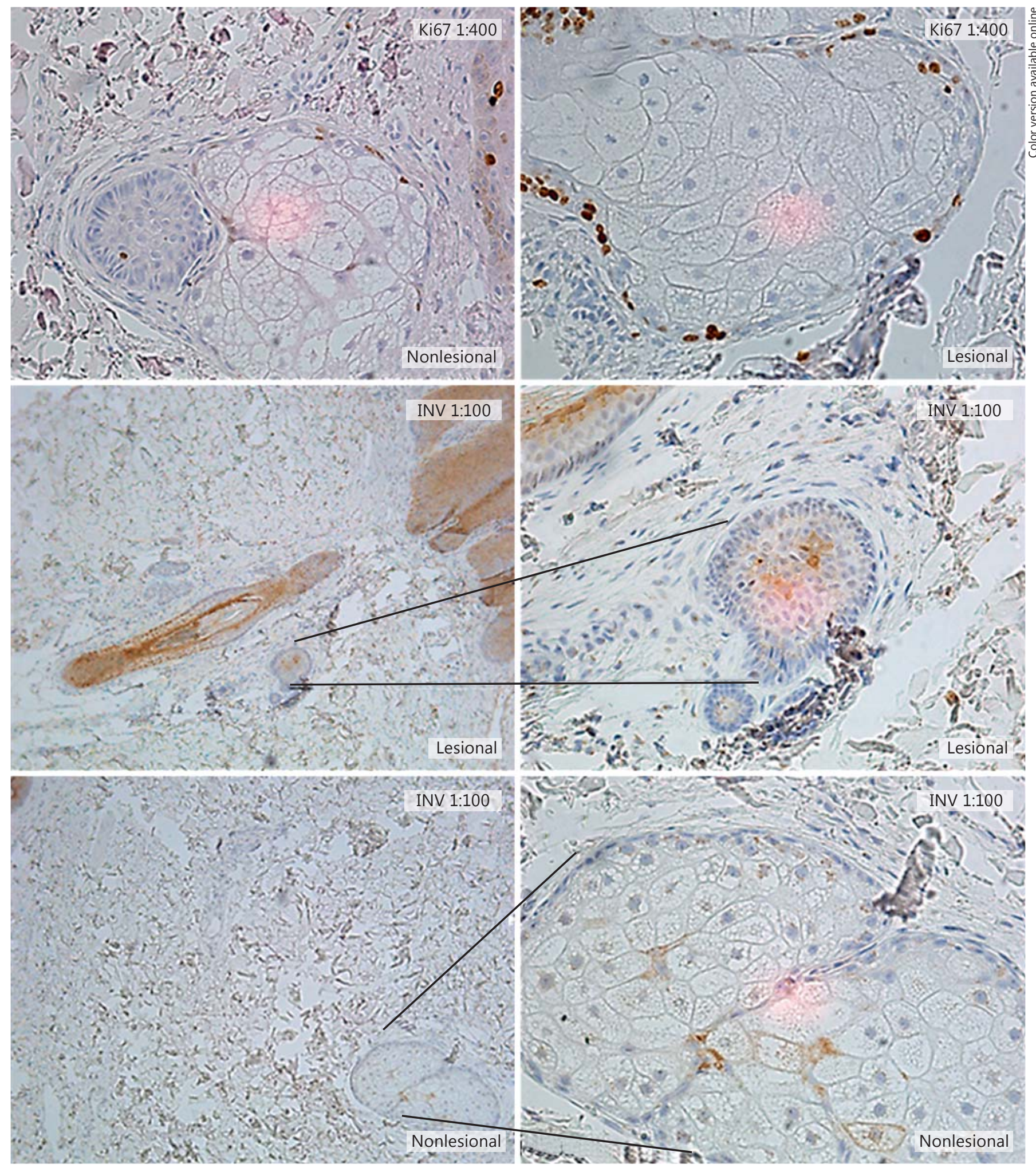

Fig. 8. Slightly higher expression of Ki67 in the lesional skin compared to the nonlesional psoriatic skin. There were unclear differences between the expression levels of involucrin (INV). This picture shows a possible elevated rate of proliferation in the lesional skin. 
barrier, and the skin immune system and its synthesis are strongly regulated by hormones, in particular by androgens. Sebocyte formation is controlled by multiple molecular pathways, such as Blimp1, Wnt, C-myc, and Hedgehog $[8,14]$. Sebaceous glands extend from the upper hair follicle and are formed from a sebocyte progenitor that expresses Blimp 1, keratin 14, and keratin 5. These cells produce a proliferative population of sebocytes that, in turn, differentiates to produce the lipid/ sebum-producing cells $[20,21]$. The derivation of sebocytes from hair follicle keratinocytes suggests the possible theory that there can exist a signal that forces the hair follicle keratinocytes to turn the normal proliferation/ differentiation process toward keratinocytes instead of sebocytes and produce more keratinocytes instead of providing sebocytes to the sebaceous gland $[13,19]$. This theory does not explain, however, where this signal of general squamous differentiation of the dermis and epidermis comes from. Furthermore, it does not provide any information about the sequence of events or whether the squamous differentiation of sebocytes comes before the acanthosis and hyperkeratosis of the epidermis or whether the pathology of the epidermis comes first and then, as a consequence, the atrophy of the sebaceous glands, or a signal, forces all parts of the skin to a squamous differentiation.

A recent study by Kamp et al. [22] on patients with hidradenitis suppurativa/acne inversa revealed a very similar result: significantly more atrophy of the sebaceous glands in the involved skin compared to that of healthy controls. This was the first stereological volume estimation of sebaceous glands in a skin disease. Braun-Falco et al. [23] have also shown an absence or decrease of sebaceous glands in a histological study of pseudopelade Brocq; however, this is a nontransient scarring disease. On the other hand, Kure et al. [24] found in a later stereological study that lobulation of the sebaceous glands was increased in androgenetic alopecia with a consequent noticeable increase of the sebaceous gland area, while the lobule volume remained unchanged, These findings are very interesting in combination with the findings of the present study in psoriasis because they raise the question of what these diseases have in common, since they both result in an excessive change of sebaceous gland volume, although the sebaceous gland is considered not to be the primary target of these disorders. At least psoriasis and hidradenitis suppurativa/acne inversa seem to have some characteristics in common, such as local hyperproliferation of keratinocytes, genetic background, chronic inflammation, and, as a consequence, good response to antitumor necrosis factor agents and other biologics and increased risk of metabolic disease as comorbidity. In addition, both smoking and increased body mass index impair the clinical picture of both diseases. However, several dermatological diseases share all or some of these characteristics. In fact, psoriasis and hidradenitis suppurativa/acne inversa are clinically very different dermatological diseases that do not even belong in the same classification subgroup. On the other hand, one could raise the question of whether it is simply that sebaceous gland atrophy occurs in all dermatological pathologies, such as when the epithelium is 'ill' and inflamed it includes the sebaceous gland. A good and simple way to confirm this is to estimate stereologically the sebaceous gland volume in different skin diseases, not only in dermatoses and chronic inflammatory diseases but also in other skin pathologies, such as epithelial skin cancer (basal cell carcinoma or squamous cell carcinoma). It would be interesting to add further comparisons with completely healthy skin in order to investigate whether the sebaceous glands are smaller in psoriasis. Such a study would entail the ethical issue of using skin biopsies from a healthy population.

The findings of this study could be a stimulus for reexamining the role of sebaceous glands in psoriasis. For example, the existing theory of psoriatic alopecia being caused by the psoriatic plaque (hyperkeratotic plaque) strangling the hair follicle can be revised for the first time. Our results suggest that psoriatic alopecia is probably developed due to a secondary abnormality of sebaceous glands rather than to the mechanical cause that has hitherto been proposed. On the other hand, however, the existence of psoriasis palmoplantaris, which selectively affects the only areas of the human body lacking hair follicles and sebaceous glands, may indicate that the involvement of sebaceous glands in psoriasis is a secondary phenomenon $[25,26]$.

In this project, the application of stereological principles in psoriasis revealed a clear reduction of sebaceous gland volume in the diseased skin. Further studies should investigate and expand these results to other skin diseases to determine whether our findings are isolated or a result of a common, more general pathology. Our findings suggest a new approach in the effort to elucidate the complicated cascade in the pathogenesis of psoriasis, and they implicate - for the first time - not only the epidermal/ dermal layers but also the appendages of this well-known yet not fully understood disease. 


\section{Statement of Ethics}

The study was approved by the Ethics Committee of the Charité - Universitätsmedizin Berlin, Freie Universität Berlin.

\section{Disclosure Statement}

The authors have no conflicts of interest to declare.

\section{References}

- 1 Headington JT, Gupta AK, Goldfarb MT, Nickoloff BJ, Hamilton TA, Ellis CN, Voorhees JJ: A morphometric and histologic study of the scalp in psoriasis. Paradoxical sebaceous gland atrophy and decreased hair shaft diameters without alopecia. Arch Dermatol 1989; 125:639-642.

2 Wilson CL, Dean D, Lane EB, Dawber RP, Leigh IM: Keratinocyte differentiation in psoriatic scalp: morphology and expression of epithelial keratins. Br J Dermatol 1994;131: 191-200.

-3 Werner B, Brenner FM, Böer A: Histopathologic study of scalp psoriasis: peculiar features including sebaceous gland atrophy. Am J Dermatopathol 2008:30:93-100.

-4 Silva CY, Brown KL, Kurban AK, Mahalingam M: Psoriatic alopecia - fact or fiction? A clinicohistopathologic reappraisal. Indian J Dermatol Venereol Leprol 2012;78:611-619.

5 Ross EK, Tan E, Shapiro J: Update on primary cicatricial alopecias. J Am Acad Dermatol 2005;53:19-37.

6 Zouboulis CC, Fimmel S, Ortmann J, Turnbull JR, Boschnakow A: Sebaceous glands; in Hoath SB, Maibach HI (eds): Neonatal Skin - Structure and Function, ed 2. New York/Basel, Marcel Dekker, 2003, pp 59-88.

-7 Zouboulis CC, Krieter A, Gollnick H, Mischke D, Orfanos CE: Progressive differentiation of human sebocytes in vitro is characterized by increased cell size and altered antigenic expression and is regulated by culture duration and retinoids. Exp Dermatol 1994;3:151-160.

8 Niemann C: Differentiation of the sebaceous gland. Dermatoendocrinol 2009;1:64-67.
9 Thiboutot D: Regulation of human sebaceous glands. J Invest Dermatol 2004;123:1-12.

10 Zouboulis CC, Chen W: The sebaceous gland and its role as an endocrine organ. World Clin Dermatol 2013;1:37-51.

11 Georgel P, Crozat K, Lauth X, Makrantonaki E, Seltmann H, Sovath S, Hoebe K, Du X, Rutschmann S, Jiang Z, Bigby T, Nizet V, Zouboulis CC, Beutler B: A TLR2-responsive lipid effector pathway protects mammals against Gram-positive bacterial skin infections. Infect Immun 2005;73:4512-4521.

12 Selleri S, Seltmann H, Gariboldi S, Shirai YF, Balsari A, Zouboulis CC, Rumio C: Doxorubicin-induced alopecia is associated with sebaceous gland differentiation. J Invest Dermatol 2006; 126:711-720.

13 Ju Q, Fimmel S, Hinz N, Stahlmann R, Xia L, Zouboulis CC: $2,3,7,8$-Tetrachlorodibenzo$p$-dioxin alters sebaceous gland cell differentiation in vitro. Exp Dermatol 2011;20:320325.

14 Zouboulis CC, Adjaye J, Akamatsu H, MoeBehrens G, Niemann K: Human skin stem cells and the ageing process. Exp Gerontol 2008;43:986-997.

15 Barrault C, Dichamp I, Garnier J, Pedretti N, Juchaux F, Deguercy A, Agius G, Bernard FX: Immortalized sebocytes can spontaneously differentiate into a sebaceous-like phenotype when cultured as a 3D epithelium. Exp Dermatol 2012;21:299-319.

16 Zouboulis CC, Xia LQ, Detmar M, et al: Culture of human sebocytes and markers of sebocytic differentiation in vitro. Skin Pharmacol 1991;4:74-83.

17 Zouboulis CC, Seltmann H, Neitzel H, Orfanos CE: Establishment and characterization of an immortalized human sebaceous gland cell line (SZ95). J Invest Dermatol 1999;113: 1011-1020.
18 Lo Celso C, Berta MA, Braun KM, Frye M, Lyle S, Zouboulis CC, Watt FM: Characterisation of bipotential epidermal progenitors derived from human sebaceous gland: contrasting roles of c-Myc and $\beta$-catenin. Stem Cells 2008;26:1241-1252.

19 Stenn KS, Zheng Y, Parimoo S: Phylogeny of the hair follicle: the sebogenic hypothesis. J Invest Dermatol 2008;128:1576-1578.

20 Gudjonsson JE, Elder JT: Psoriasis: epidemiology. Clin Dermatol 2007;25:535-546.

21 Krueger G, Ellis N: Psoriasis-recent advances in understanding its pathogenesis and treatment. J Am Acad Dermatol 2005;53:94-100.

22 Kamp S, Fiehn AM, Stenderup K, Rosada C, Pakkenberg B, Kemp K, Dam TN, Jemec GB: Hidradenitis suppurativa: a disease of the absent sebaceous gland? Sebaceous gland number and volume are significantly reduced in uninvolved hair follicles from patients with hidradenitis suppurativa. Br J Dermatol 2011; 164:1017-1022.

23 Braun-Falco O, Imai S, Schmoeckel C, Steger O, Bergner T: Pseudopelade of Brocq. Dermatologica 1986;172:18-23.

24 Kure K, Isago T, Hirayama T: Changes in the sebaceous gland in patients with male pattern hair loss (androgenic alopecia). J Cosmet Dermatol 2015;14:178-184.

25 Stenn KS, Karnik P: Lipids to the top of hair biology. J Invest Dermatol 2010;130:12051207.

26 Morsy H, Kamp S, Thrane L, Behrendt N, Saunder B, Zayan H, Elmagid EA, Jemec GB: Optical coherence tomography imaging of psoriasis vulgaris: correlation with histology and disease severity. Arch Dermatol Res 2010; 302:105-111. 